\title{
SNAIL expression correlates with the translocation of syndecan-1 intracellular domain into the nucleus in prostate cancer cell lines
}

\author{
NANCY FARFÁN ${ }^{1}$, OCTAVIO ORELLANA-SERRADELL ${ }^{1}$, DANIELA HERRERA $^{1}$, \\ DOMINIQUE CHRZANOWSKY ${ }^{1}$, PAULINA CUBILLOS ${ }^{1}$, GABRIEL MARÍN $^{1}$, \\ ANTONIO GARCÍA DE HERREROS ${ }^{2,3}$, ENRIQUE A. CASTELLÓN ${ }^{1}$ and HÉCTOR R. CONTRERAS ${ }^{1}$ \\ ${ }^{1}$ Department of Basic and Clinical Oncology, Faculty of Medicine, University of Chile, Santiago 8380453, Chile; \\ ${ }^{2}$ Program of Research in Cancer, Institute of Medical Research Hospital of Mar, 08003 Barcelona; \\ ${ }^{3}$ Department of Experimental Sciences and Health, University of Pompeu Fabra, 08002 Barcelona, Spain
}

Received September 16, 2019; Accepted January 21, 2020

DOI: $10.3892 / \mathrm{ijmm} .2020 .4488$

\begin{abstract}
Zinc finger protein SNAI1 (SNAIL) and zinc finger protein SNAI2 (SLUG) transcription factors promote epithelial-mesenchymal transition, a process through which epithelial cells acquire a mesenchymal phenotype, increasing their migratory and invasive properties. In prostate cancer (PCa) progression, increased expression levels of SNAIL and SLUG have been described. In advanced PCa, a decrease in the cell surface proteoglycan syndecan-1 (SDC-1), which has a role in cell-to-extracellular matrix adhesion, has been observed. Notably, SDC-1 nuclear location has been observed in mesenchymal cancers. The present study aimed to determine if SNAIL and SLUG may be associated with the nuclear location of SDC-1 in PCa. To determine the location of SDC-1, antibodies against its intracellular domain (ID) or extracellular domain (ED) were used in benign prostatic hyperplasia (BPH) and PCa samples with high Gleason scores. Only ID-SDC-1 was located in the cell nuclei in advanced PCa samples, but not in the BPH samples. ED-SDC-1 was located in the cell membrane and cytoplasm, exhibiting decreased levels in $\mathrm{PCa}$ in comparison with those in BPH. Furthermore, LNCaP and PC3 PCa cell lines with ectopic SNAIL expression exhibited nuclear ID-SDC-1. No change was observed in the ED-SDC-1 levels, and maintained its location in the cell membrane and cytoplasm. SLUG induced no change in ID-SDC-1 location. At the protein level, an association between SNAIL and nuclear ID-SDC-1 was observed. In conclusion, the results of the
\end{abstract}

Correspondence to: Dr Héctor R. Contreras or Dr Enrique A. Castellón, Department of Basic and Clinical Oncology, Faculty of Medicine, University of Chile, Independencia 1027, Santiago 8380453, Chile

E-mail: hcontrer@med.uchile.cl

E-mail: ecastell@med.uchile.cl

Key words: prostate cancer, syndecan-1 intracellular domain, nuclear location, zinc finger protein SNAI1, zinc finger protein SNAI2 present study demonstrated that nuclear ID-SDC-1 localization was associated with SNAIL expression in PCa cell lines.

\section{Introduction}

Prostate cancer (PCa) is the second most commonly diagnosed cancer in men and the fifth most common cause of cancer-associated mortality worldwide (1). PCa progression involves transformation of the prostate gland structure. During this process, which is known as epithelial-mesenchymal transition (EMT), epithelial cells lose their characteristics, such as cell-to-extracellular matrix (ECM) adhesion, and increase their migratory and invasive properties, acquiring a mesenchymal phenotype $(2,3)$. This process has been associated with an increase in EMT transcription factors, including the zinc finger protein SNAI1 (SNAIL), Twist-related protein (TWIST) and zinc finger E-box-binding (ZEB) families, which repress epithelial markers expression (4).

PCa progression has been associated with increases in the levels of SNAIL and SLUG, which are SNAIL family members, and TWIST transcription factors (5), while the levels of epithelial cadherin (E-cadherin) and other epithelial markers such as syndecan-1 (SDC-1) decrease following PCa progression (5-7). In this context, ectopic SDC-1 expression has been associated with decreased rates of tumor growth in myeloma (8), breast cancer (9) and PCa (10).

SDC-1 is a transmembrane proteoglycan primarily expressed in epithelial cells, with a role in cell-to-ECM adhesion, motility and intracellular signalling of other receptors, such as integrins. The extracellular domain of SDC-1 (ED-SDC-1) is a large fragment with glycosaminoglycans [heparan sulfate (HS) and chondroitin sulfate], which binds extracellular ligands. The transmembrane domain is connected to the intracellular domain of SDC-1 (ID-SDC-1), which has a smaller extension (11).

Although SDC-1 has a cellular membrane location, previous studies have described nuclear SDC-1 location in malignant mesothelioma cells $(12)$, myeloma cells $(13,14)$ and mesenchymal tumors $(15,16)$. Also, shed ED-SDC-1 has been identified in the nucleus of bone marrow-derived stromal cells (17). In these articles, HS has an important role in nuclear traffic $(13,15,17-19)$. 
The function of nuclear SDC-1 is not clear; however, histone acetyltransferase (HAT) inhibition, leading to chromatin compaction (13), cell cycle control, decreases in proliferation, transcriptional machinery regulation and protein transport to the nucleus (19), have been suggested. Additionally, our previous study demonstrated that SDC-1 expression was repressed by ZEB1 in prostate cell lines (20). However, an association between SNAIL family transcription factors and nuclear SDC-1 location has not been demonstrated yet.

Based on these data, the present study aimed to investigate if SNAIL or SLUG may be associated with the nuclear location of SDC-1 in PCa.

\section{Materials and methods}

Specimens. Samples of benign prostatic hyperplasia $(\mathrm{BPH})(\mathrm{n}=3)$ and those with high Gleason Score PCa (8 and 9) $(\mathrm{n}=3)$, were obtained from biopsy archives of the Anatomy and Pathology Service, Clinical Hospital of the University of Chile (CHUCh). All protocols and authorization for biopsy use were approved by the Faculty of Medicine and CHUCh ethics committees (approval no. 135-2015). These protocols included written informed consent of the patients in order to use part of the tumor samples for research purposes. All protocols and handling of hazardous materials were approved by the Faculty of Medicine of the University of Chile Risk and Biosecurity Unit.

Immunohistochemistry. The immunohistochemical procedures and digitalization of the images (magnification, $\mathrm{x} 20$ ) were performed as described previously (20). The primary antibodies were as follows: Anti-SNAIL (1:100; cat. no. 3879; Cell Signaling Technology, Inc.); anti-SLUG (1:50; cat. no. sc-15391; Santa Cruz Biotechnology, Inc.); anti-ED-SDC-1 (1:100; cat. no. sc-5632; Santa Cruz Biotechnology, Inc.); and anti-ID-SDC-1 (1:100; cat. no. 362900, Invitrogen; Thermo Fisher Scientific, Inc.). ImageJ v.1.52f software [National Institutes of Health (NIH)] was used to quantify the images. For each immunodetection, 50 images were included and quantified.

Cell culture. The human PCa LNCaP (CRL-1740 ${ }^{\mathrm{TM}}$ ) and PC3 (CRL-1435 ${ }^{\mathrm{TM}}$ ) cell lines were obtained from the American Type Culture Collection and cultured as previously described (20).

Lentiviral transduction. Transduction was performed as described in a previous study (20), with lentiviral particles purchased from GenTarget Inc. and the lentiviral plasmid pLenti suCMV (target sequence)-Rsv red fluorescent protein (RFP)-Puro (GenTarget Inc.), in which the target sequences were SNAIL (NM_005985.3) or SLUG (NM_003068.4), or without a target sequence as the empty vector $(\mathrm{EV})$ control.

Immunofluorescence. A total of $5 \times 10^{4}$ cells were seeded on coverslips in 24-well plates. The procedure was performed as previously described (21). The primary antibodies dilutions were: 1:50 for anti-ID-SDC-1 (cat. no. 362900; Invitrogen; Thermo Fisher Scientific, Inc.); 1:100 for anti-ED-SDC-1 (cat. no. sc-5632; Santa Cruz Biotechnology, Inc.); and 1:100 for anti-CD44 antigen (CD44; cat. no. ab6124; Abcam). The fluorophores conjugated to the secondary antibodies were
Alexa Fluor 488 and Alexa Fluor 405 (cat. nos. A-11008 and A-31553, respectively; both from Thermo Fisher Scientific, Inc.; 1:200). The mounted coverslips were observed under a confocal microscope (LSM-410 Axiovert 100 + Axio Imager; Carl Zeiss AG; magnification, x600). Positive RFP expression was used as the marker of successful transduction. In total, 50 cells were quantified for each marker. To determine only nuclear ID-SDC-1, Adobe Photoshop CS6 Software (2012, version 13.0; Adobe Systems, Inc.) was utilized to delete the nuclei from the DAPI images, which were overlapped with the ID-SDC-1 images. Quantification and the Menders' overlap coefficient were determined using ImageJ v.1.52f software (NIH).

Total, cytoplasmic and nuclear protein extraction. Cells were seeded in a $100-\mathrm{mm}$ dish $\left(3 \times 10^{6}\right.$ or $2.2 \times 10^{6}$ for $\mathrm{LNCaP}$ or PC3 cells, respectively). Total protein extraction was performed as previously described (20). For cytoplasmic and nuclear protein extraction, cells were harvested, treated with $300 \mu \mathrm{l}$ buffer 1 [50 mM Tris, $0.5 \%$ Triton X-100, $137 \mathrm{mM} \mathrm{NaCl}, 10 \%$ glycerol and protease inhibitors (Roche Diagnostics)] and incubated for $15 \mathrm{~min}$ on ice. The extracts were centrifuged at $500 \mathrm{x} \mathrm{g}$ for $15 \mathrm{~min}$ at $4^{\circ} \mathrm{C}$; these supernatants contained the cytoplasmic proteins. The pellet was then resuspended in $150 \mu \mathrm{l}$ buffer 1 (50 mM Tris $\mathrm{pH} 7.5,0,5 \%$ Triton X-100, $137 \mathrm{mM} \mathrm{NaCl}, 10 \%$ glycerol + protease and phosphatase inhibitors) with $0.5 \%$ SDS, and then passed through a tuberculin syringe $(27.5 \mathrm{G}$ x 1/2"; Plastipak ${ }^{\mathrm{TM}}$; BD Biosciences), sonicated at $20 \mathrm{kHz}$ for $10 \mathrm{sec}$ and centrifuged at $17,000 \mathrm{x} \mathrm{g}$ for $15 \mathrm{~min}$ at $4^{\circ} \mathrm{C}$. Following this step, the supernatant now contained the nuclear proteins. A BCA kit (Thermo Fisher Scientific, Inc.) was used for protein quantification.

Western blot analysis. SDS-PAGE analysis was performed following loading of $50 \mu \mathrm{g}$ cytoplasmic or total protein and $10 \mu \mathrm{g}$ nuclear protein into each lane. The gels use were $6-12 \%$. The proteins were then transferred to a nitrocellulose membrane and blocked with $5 \%$ milk in $1 \mathrm{X}$ TBS $/ 0.1 \%$ Tween-20 at room temperature for $1 \mathrm{~h}$. The membranes were incubated with anti-ED-SDC-1 (1:500; cat. no. sc-5632; Santa Cruz Biotechnology, Inc.), ID-SDC-1 (1:250; cat. no. sc-7099; Santa Cruz Biotechnology, Inc.), lamin-B1 (1:1,000; cat. no. sc-374015; Santa Cruz Biotechnology, Inc.), $\beta$-actin (1:1,000; cat. no. sc-81178; Santa Cruz Biotechnology, Inc.), SNAIL (1:1,000; cat. no. C15D3; Cell Signaling Technology, Inc.) and SLUG (1:1,000; cat. no. C19G7; Cell Signaling Technology, Inc.), vimentin (1:500; cat. no. ab8978; Abcam) and E-cadherin (1:1,000; cat. no. 610181; BD Transduction Laboratories; BD Biosciences) primary antibodies overnight at $4^{\circ} \mathrm{C}$. The membranes were then incubated with the following horseradish peroxidase (HRP)-conjugated secondary antibodies for $1 \mathrm{~h}$ at room temperature: Peroxidase AffiniPure Goat Anti-Mouse IgG (H+L) (cat. no. 115-035-003), Peroxidase AffiniPure Goat Anti-Rabbit IgG (H+L) (catalog no. 111-035-003) and Peroxidase AffiniPure Rabbit Anti-Goat IgG (H+L) (catalog no. 305-035-045), all purchased from Jackson ImmunoResearch Laboratories, Inc. and all used at 1:10,000. The membranes were developed using the Pierce ${ }^{\mathrm{TM}}$ Enhanced chemiluminescence Western Blotting Detection kit for HRP (cat. no. 32209; Thermo Fisher Scientific, Inc.) in an 
A
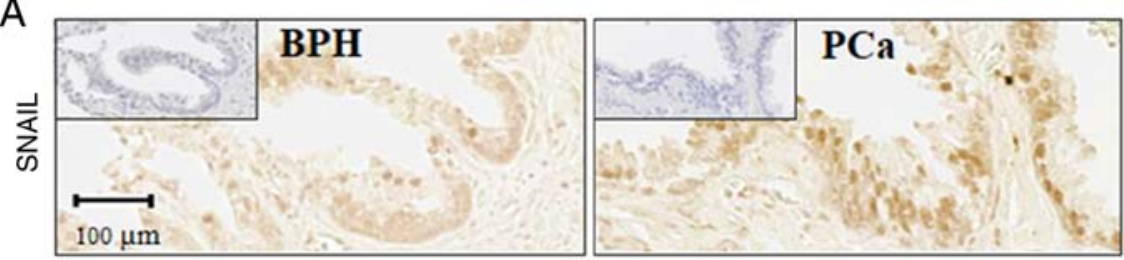

C

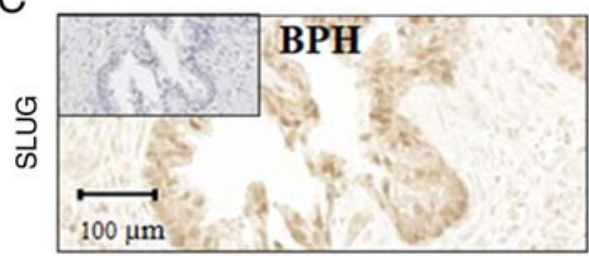

E

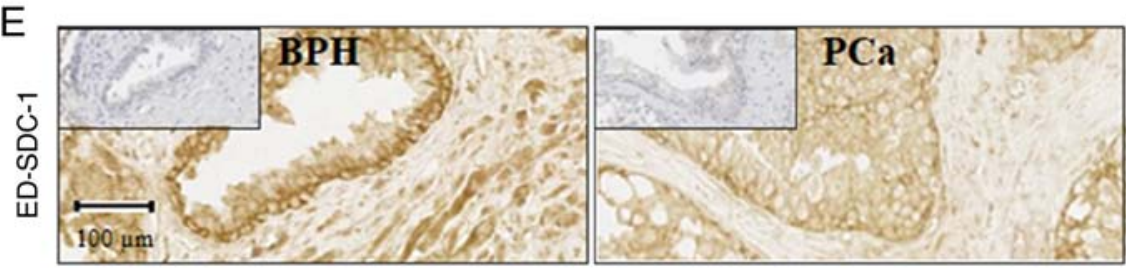

G

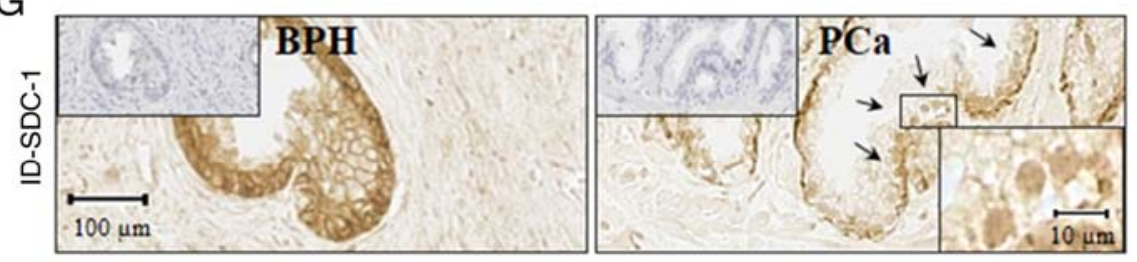

B

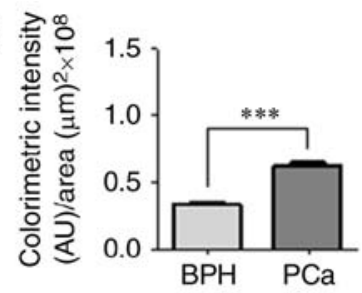

D

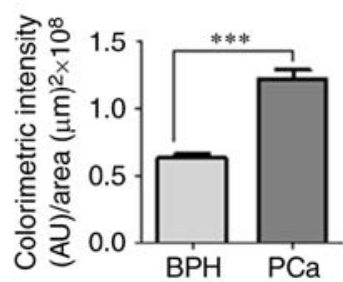

$\mathrm{F}$
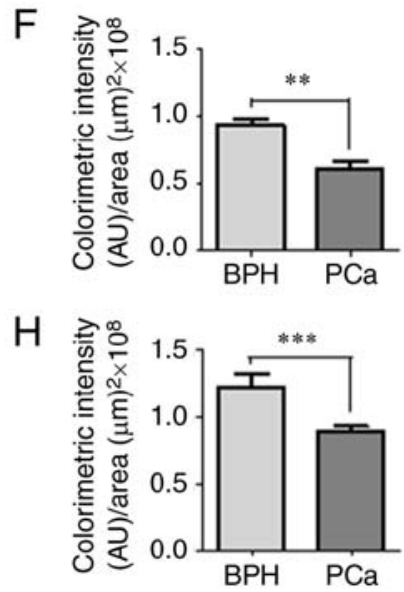

Figure 1. Immunohistochemistry in benign prostatic hyperplasia and prostate cancer samples. Localization of (A) SNAIL, (C) SLUG, (E) ED-SDC-1 and (G) ID-SDC-1. (G) Nuclear ID-SDC-1 (black arrows) and magnification (rectangle in the center of the image) are included in the lower right corner. Hematoxylin staining (negative control) is presented in the upper left corner. (B) SNAIL ( $\mathrm{P}=0.0011)$, (D) SLUG (P=0.0004), (F) ED-SDC-1 (P=0.0011) and (H) ID-SDC-1 $(\mathrm{P}=0.0004)$ protein levels were quantified. The data represent the average of 3 independent experiments, and the data are presented as the mean \pm standard error of the mean. Data were analyzed using a Student's t-test. ${ }^{* *} \mathrm{P}<0.01,{ }^{* * *} \mathrm{P}<0.001$. SNAIL, zinc finger protein SNAI1; SLUG, zinc finger protein SNAI2; SDC-1, syndecan-1; ED, extracellular domain; ID, intracellular domain.

automatic system (Fusion FX5-XT; Vilber Lourmat Sté) and quantified using ImageJ $1.52 \mathrm{f}$ software (NIH).

Statistical analysis. The data are presented as the mean \pm standard error of the mean. A one-way analysis of variance for repeated measurements was used to analyze statistical significance, followed by Tukey's post hoc test. Student's t-test was used to compare continuous variables between two groups. $\mathrm{P}<0.05$ was considered to indicate a statistically significant difference. Analyses were performed using GraphPad Prism 5 (GraphPad Software, Inc.).

\section{Results}

SNAIL, SLUG and ED-SDC-1 exhibit altered expression levels in PCa samples. In the BPH samples, SNAIL and SLUG exhibited weak and primarily nuclear immunoreactivity. However, in the high Gleason score PCa samples, an increase in intensity was observed in the nuclei of epithelial glandular cells and in the number of positively stained nuclei. These observations were similar to data from previous studies, where SNAIL and SLUG levels increased according to disease progression (5). In the high Gleason score samples, certain cells exhibited cytoplasmic staining, which may be associated with the tissue disorganization in this PCa stage (Fig. 1A-D). In BPH, ED-SDC-1 was located in the membrane of epithelial cells in the basolateral region and more intensely in the glandular basal zone (Fig. 1E). In the high Gleason score samples, ED-SDC-1 expression decreased in comparison with that of BPH samples (Fig. 1E and F). These observations are in agreement with previously published data (5-7).

ID-SDC-1 is located in the nucleus of PCa samples. In the high Gleason score PCa samples, ID-SDC-1 was identified in the nuclei of epithelial cells, in addition to the classical cell membrane location described for SDC-1 (Fig. 1G). In addition, in the BPH samples, ID-SDC-1 was only located superficially, like ED-SDC-1 (Fig. 1G). Total ID-SDC-1 levels were decreased in high Gleason score $\mathrm{PCa}$ in comparison with those in BPH samples (Fig. 1H).

Ectopic SNAIL expression is correlated with ID-SDC-1 location in the nuclei of PCa cell lines. To determine if SNAIL and SLUG could be associated with nuclear ID-SDC-1 location, ID-SDC-1 was analyzed in LNCaP and PC3 PCa cell lines with SNAIL or SLUG ectopic expression. SNAIL 
A
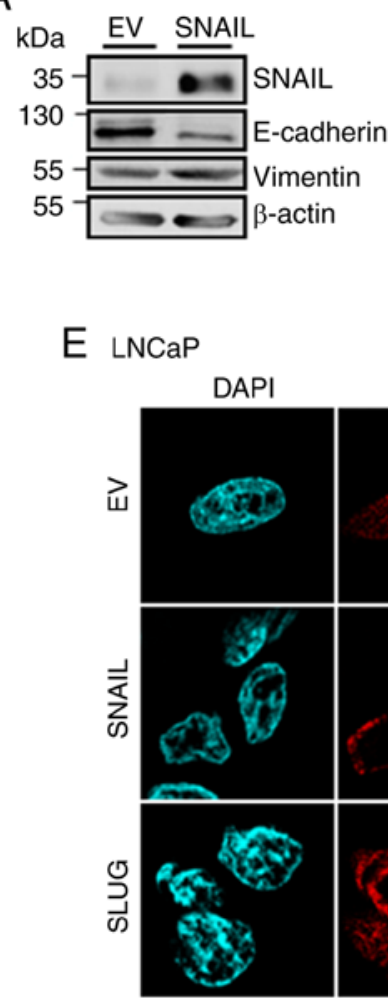

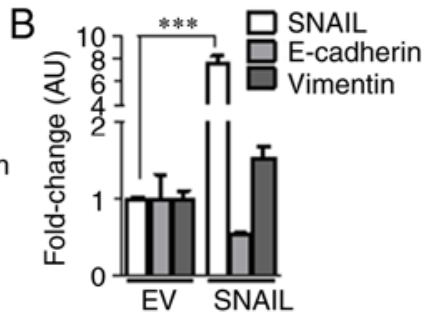

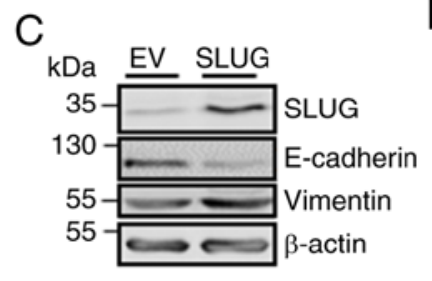

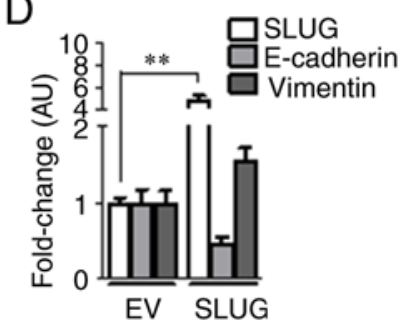

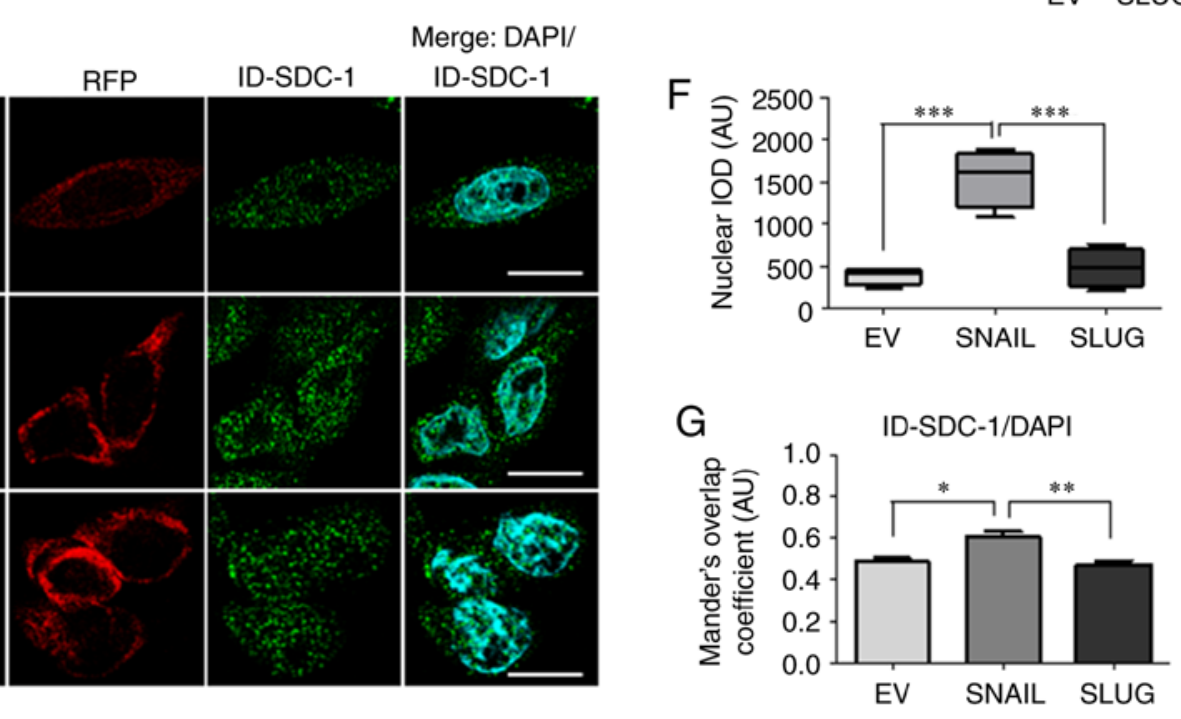

H LNCaP
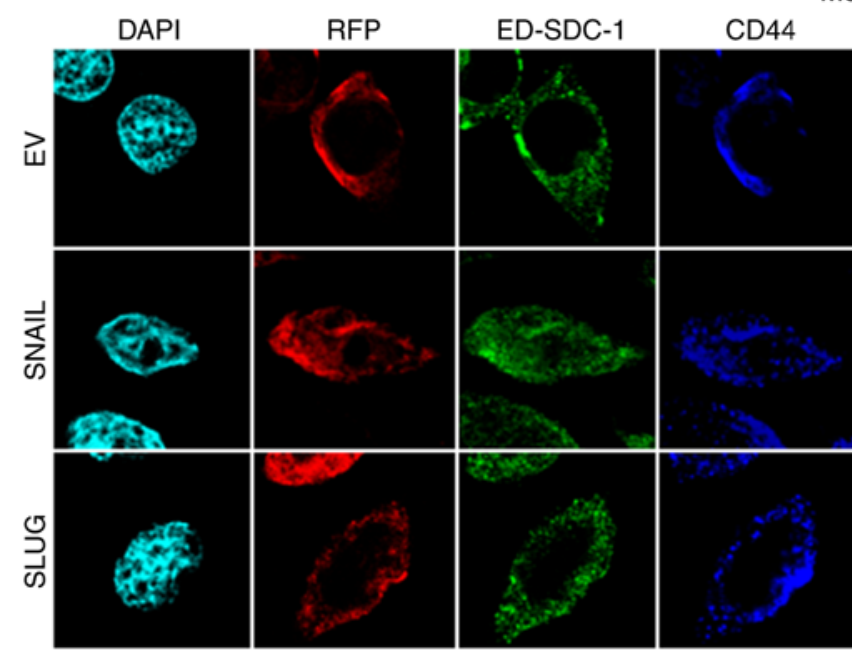

Merge: ED-SDC-1/
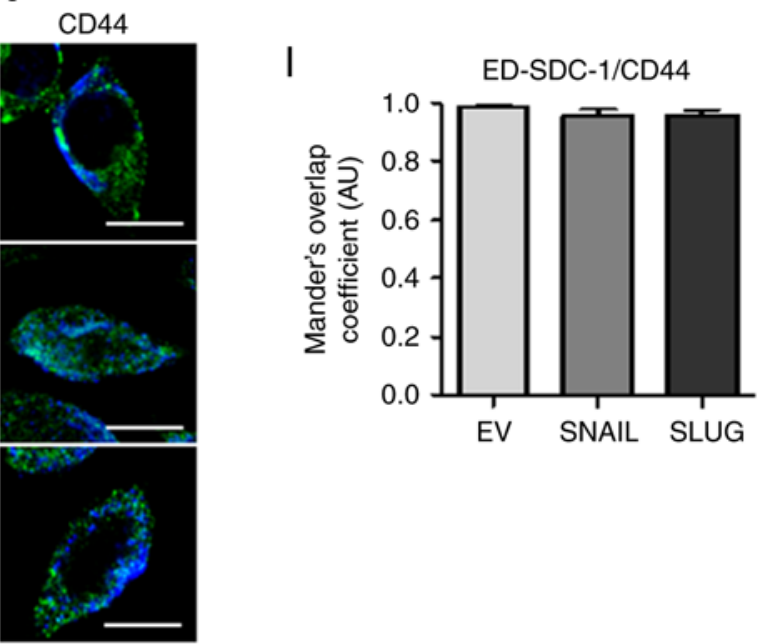

Figure 2. ID-SDC-1 and ED-SDC-1 location in LNCaP cells with ectopic SNAIL or SLUG expression. (A and C) Western blot analysis of SNAIL, SLUG, vimentin and E-cadherin protein levels. (B and D) Quantification of the western blot analysis data. Data were analyzed using a Student's t-test. (E) Confocal microscopy of DAPI (nuclei), RFP (transduction control) and ID-SDC-1 (green) in EV, SNAIL or SLUG-transduced cells. (F) Nuclear ID-SDC-1 quantification (integrated optical density per area, arbitrary units). Data were analyzed using ANOVA followed by a Tukey post hoc test. (G) Colocalization of ID-SDC-1 with DAPI was assessed using Manders' overlap coefficient. Data were analyzed using analysis of variance followed by a Tukey post hoc test. (H) Confocal microscopy of DAPI (nuclei), RFP (transduction control), ED-SDC-1 (green) and CD44 (blue) in EV, SNAIL or SLUG-transduced cells. Scale bar=10 $\mu$ m. (I) Colocalization of ED-SDC-1 with CD44 was assessed using Manders' overlap coefficient. Data were analyzed using ANOVA followed by a Tukey post hoc test. The data represent the average of 3 independent experiments, and the data are presented as the mean \pm standard error of the mean. ${ }^{*} \mathrm{P}<0.05,{ }^{* *} \mathrm{P}<0.01$, ${ }_{* * * *} \mathrm{P}<0.001$. SDC-1, syndecan-1; ED, extracellular domain; ID, intracellular domain; SNAIL, zinc finger protein SNAI1; SLUG, zinc finger protein SNAI2; EV, empty vector; RFP, red fluorescent protein; ANOVA, analysis of variance; CD44, CD44 antigen.

and SLUG efficiency transduction data are presented in Figs. 2A-D and 3A-D, respectively, in addition to changes in the mesenchymal marker vimentin and the epithelial marker E-cadherin (Figs. 2A-D and 3A-D, respectively).

Nuclear ID-SDC-1 levels were evaluated in the DAPI-delimited region.In the EV cells, ID-SDC-1 was located in the cytoplasm and nucleus (Figs. 2E and 3E). Nuclear ID-SDC-1 levels were increased in cells with ectopic SNAIL expression. Ectopic SLUG expression induced no change in nuclear ID-SDC-1 levels with respect to that of EV cells (Figs. 2E, F and 3E-G). In the SNAIL-transduced cells, nuclear ID-SDC-1 exhibited a dotted fluorescence pattern (Figs. 2E and 3E), which 
A

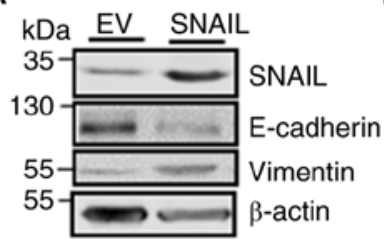

$E$

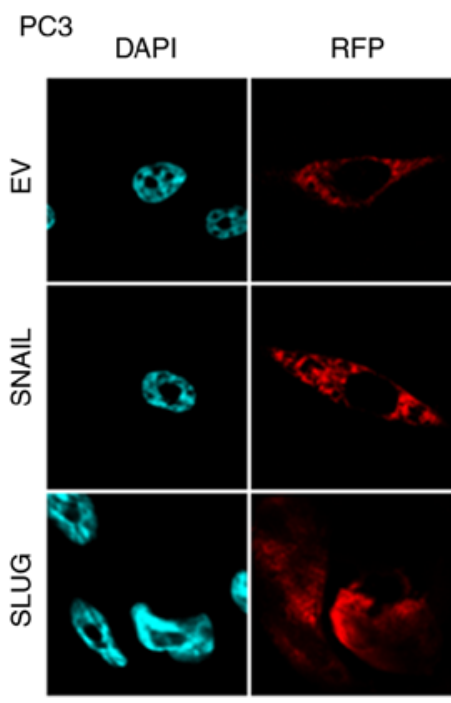

G

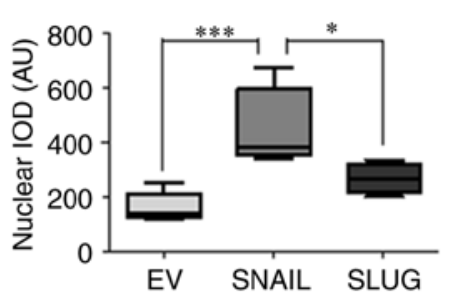

C

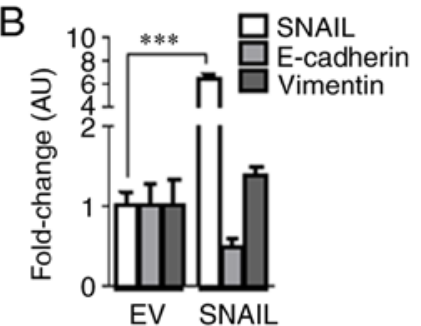

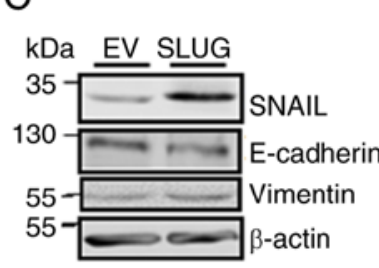

D

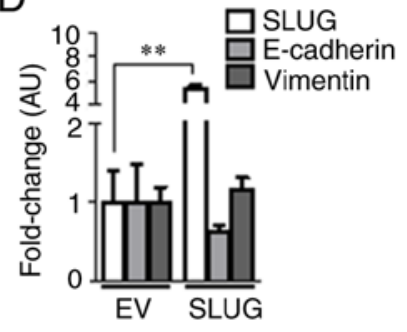

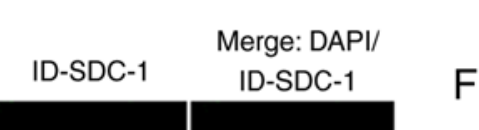

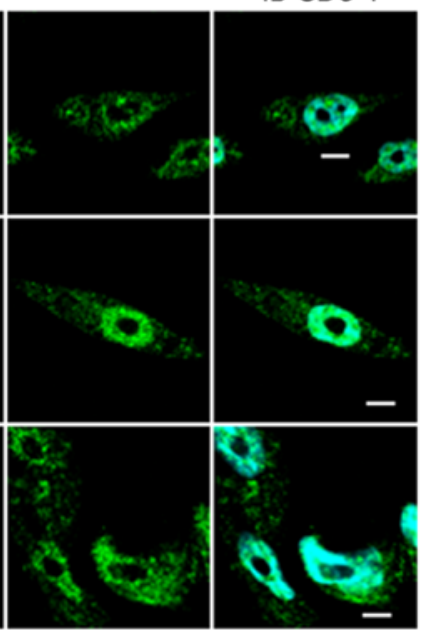

PC3

PC3 DAPI Merge: DAPI/
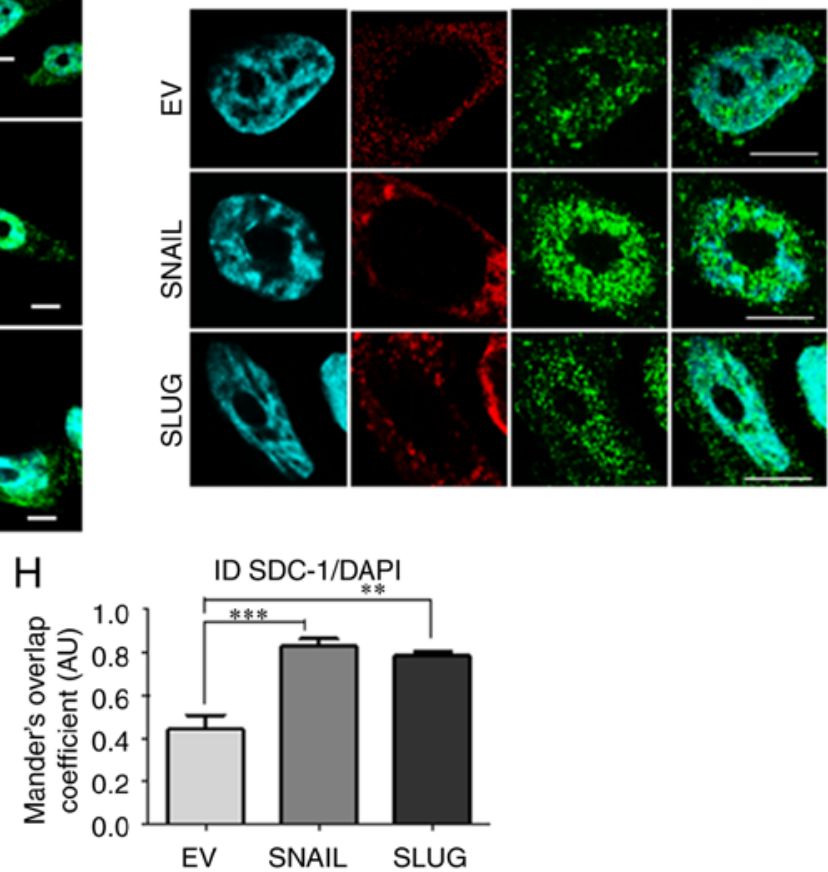

I PC3

DAPI

RFP

ED-SDC-1

Merge: ED-SDC-1/
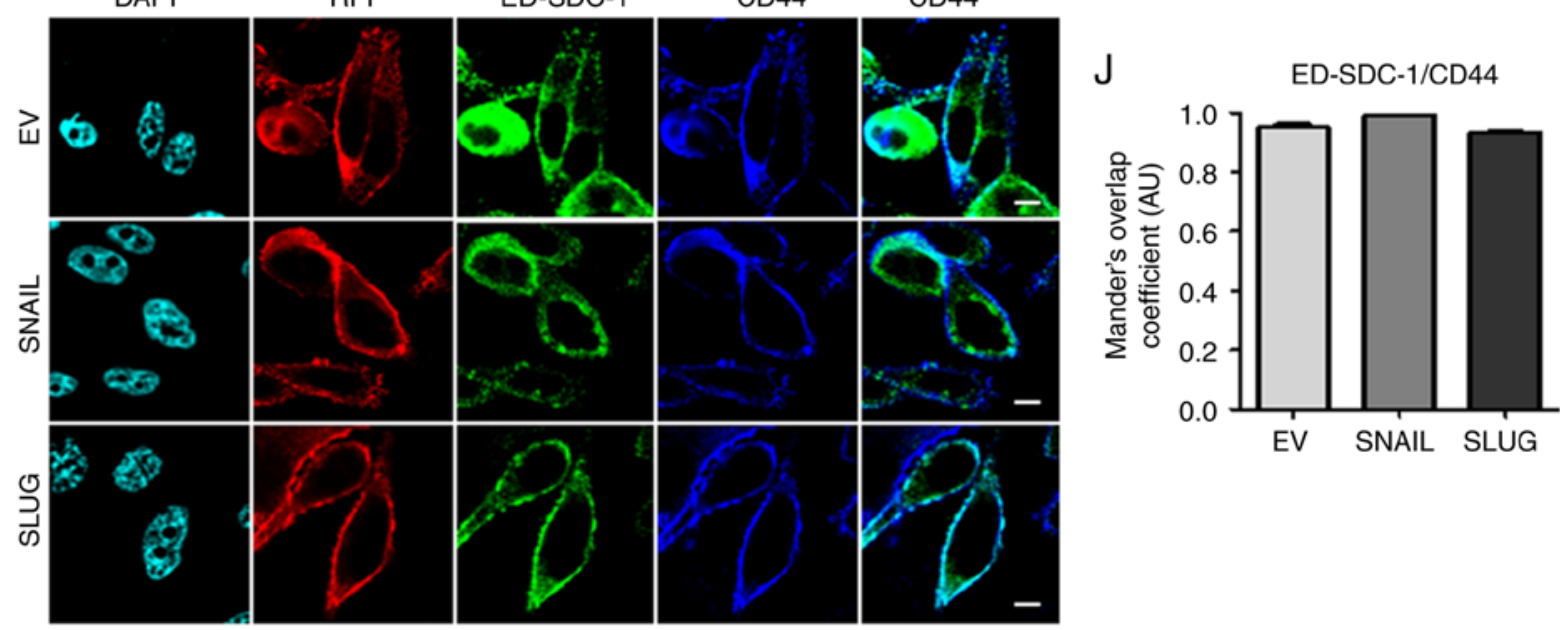

Figure 3. ID-SDC-1 and ED-SDC-1 location in PC3 cells with ectopic SNAIL or SLUG expression. (A and C) Western blot analysis of SNAIL, SLUG, vimentin and E-cadherin protein levels. (B and D) Quantification of the western blot analysis data. Data were analyzed using a Student's t-test. (E) Confocal microscopy of DAPI (nuclei), RFP (transduction control) and ID-SDC-1 (green) in EV, SNAIL or SLUG cells. Scale bar=10 $\mu$ m. (F) Nuclear region magnification. Scale bar $=10 \mu \mathrm{m}$. (G) Nuclear ID-SDC-1 quantification (integrated optical density per area, arbitrary units). Data were analyzed using ANOVA followed by a Tukey post hoc test. (H) ID-SDC-1 with DAPI Manders' overlap coefficient. Data were analyzed using ANOVA followed by a Tukey post hoc test. (I) Confocal microscopy of DAPI (nuclei), RFP (transduction control), ED-SDC-1 (green) and CD44 (blue) in the EV, SNAIL or SLUG cells. Scale bar=10 $\mu$ m. (J) ED-SDC-1 with CD44 Manders' overlap coefficient. Data were analyzed using ANOVA followed by a Tukey post hoc test. The data represent the average of three independent experiments and are presented as the mean \pm standard error of the mean. ${ }^{*} \mathrm{P}<0.05,{ }^{* *} \mathrm{P}<0.01$, ${ }^{* * *} \mathrm{P}<0.001$. SDC-1, syndecan-1; ED, extracellular domain; ID, intracellular domain; SNAIL, zinc finger protein SNAI1; SLUG, zinc finger protein SNAI2; EV, empty vector; RFP, red fluorescent protein; ANOVA, analysis of variance. 

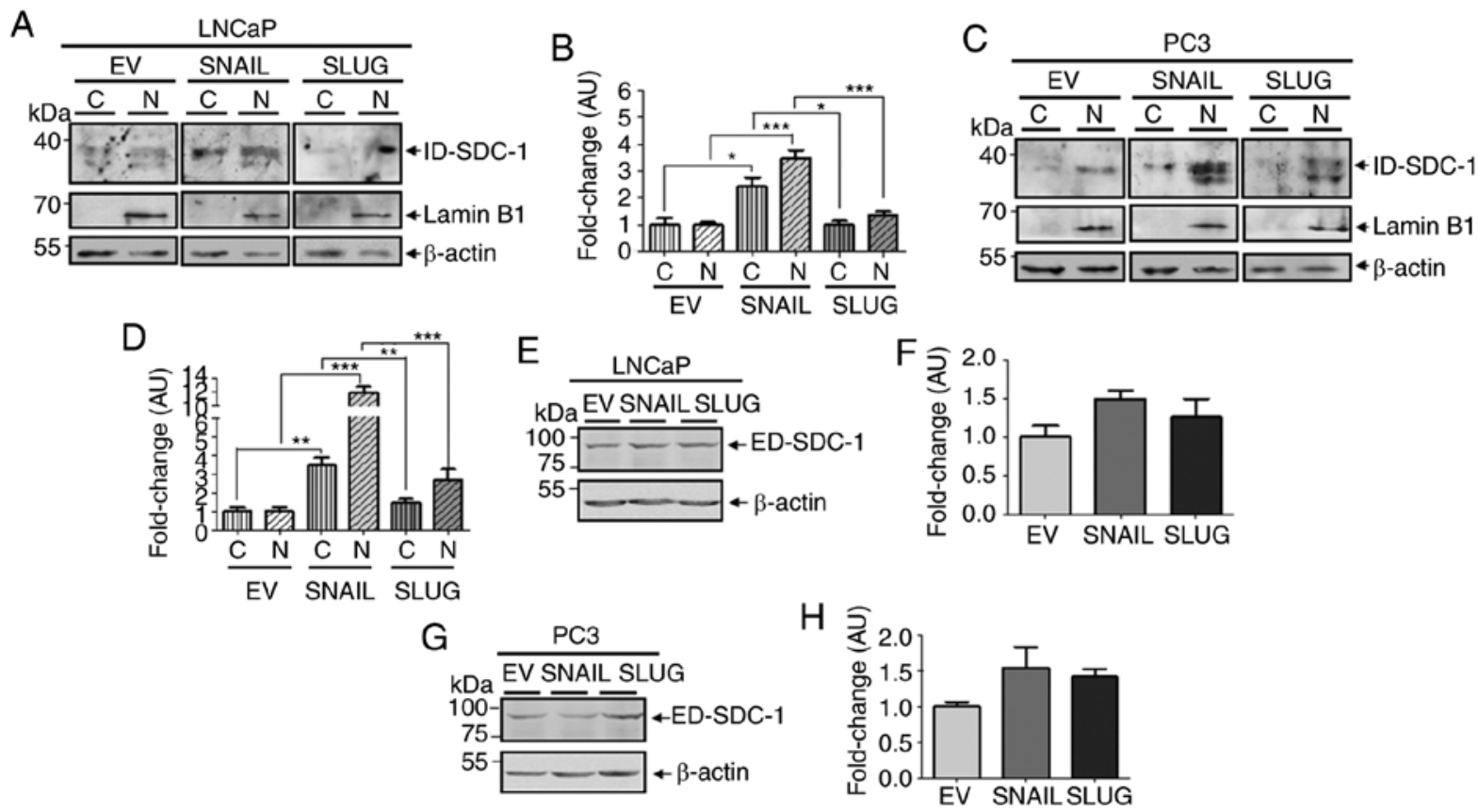

Figure 4. ID-SDC-1 and ED-SDC-1 protein levels in the cytoplasm and nucleus of LNCaP and PC3 cells with ectopic SNAIL or SLUG expression. Nuclear and cytoplasmic ID-SDC-1 protein levels of (A and B) LNCaP and (C and D) PC3 cells with ectopic EV, SNAIL or SLUG expression. Total ED-SDC-1 protein levels in (E and F) LNCaP or (G and H) PC3 cells. The levels were noramlized to those of lamin B1 (nuclear proteins) and $\beta$-actin (cytoplasmic and total proteins). The fold-change (arbitrary units) was normalized to (B and F) EV LNCaP and (D and H) PC3 protein levels. Data were analyzed using ANOVA followed by a Tukey post hoc test. The data represent the average of 3 independent experiment, and the data are presented as the mean \pm standard error of the mean. ${ }^{*} \mathrm{P}<0.05,{ }^{* *} \mathrm{P}<0.01,{ }^{* * *} \mathrm{P}<0.001$. SDC-1, syndecan-1; ED, extracellular domain; ID, intracellular domain; EV, empty vector; SNAIL, zinc finger protein SNAI1; SLUG, zinc finger protein SNAI2.

can be clearly observed in the magnified images of ID-SDC-1 in PC3 cells (Fig. 3F). Nuclear ID-SDC-1 was observed in regions with and without DAPI staining. In cells with ectopic SNAIL expression, increased ID-SDC-1 levels were observed with DAPI co-localization (Figs. 2G and 3H). PC3 cells with ectopic SLUG expression exhibited higher nuclear ID-SDC-1 levels with DAPI co-localization (Fig. $3 \mathrm{H}$ ), whereas this was not observed in LNCaP cells (Fig. 2G).

ED-SDC-1 maintained its location in the membrane and cytoplasm of LNCaP and PC3 cells (Figs. 2Hand 3I). ED-SDC-1 was co-localized with the surface marker CD44, with similar results in all the transductions (Figs. 2I and 3J).

SNAIL induces an increase in ID-SDC-1 levels in the cell nucleus and cytoplasm. To determine whether the increase in ID-SDC-1 levels observed was only in the nucleus or in the nucleus and the cytoplasm of cells, the protein levels were determined in LNCaP and PC3 cells with SNAIL or SLUG ectopic expression (Fig. 4A-D). Ectopic SNAIL expression increased the nuclear levels of ID-SDC-1 and, to a decreased level, the cytoplasm levels in LNCaP and PC3 cells (Fig. 4A-D). Ectopic SLUG expression induced no change in ID-SDC-1 levels in either of the PCa cell lines analyzed (Fig. 4A-D). ED-SDC-1 total protein levels were similar in EV, SNAIL and SLUG-overexpressing LNCaP and PC3 cells (Fig. 4E-H).

\section{Discussion}

The present study demonstrated the nuclear location of ID-SDC-1 in PCa samples. Absence of ED-SDC-1 in the nucleus may be a particular feature of PCa, as SDC-1 has been observed in the nucleus of cells (13-18) and HS proteoglycans are involved in nuclear traffic $(13,15,17,18)$. Nevertheless, ID-SDC-1, which lacks HS, may be translocated to the nucleus through its positively charged amino acidic sequence (RMKKK), which could be identified as a nuclear location sequence (15). Therefore, a mutation in this amino acid sequence may eliminate this possibility in PCa cells.

ID-SDC-1 production may be the consequence of juxtamembrane intracellular domain shedding, which could be performed by $\gamma$-secretase (22), or an alternative translation initiation, as described for the human epidermal growth receptor (HER2) intracellular domain (23). Both potential explanations should be investigated in future studies.

Nuclear ID-SDC-1 location was observed in EV LNCaP or PC3 cells in the present study. This may be due to the metastatic origin of these cells, which must have undergone EMT and a mesenchymal-epithelial transition to establish a metastatic niche in a distant organ.

The levels of ID-SDC-1 nuclear location were significantly increased in the presence of ectopic SNAIL expression in PC3 cells compared with in LNCaP cells. ID-SDC-1 was observed in nuclear regions with and without DAPI staining. Nuclear regions without DAPI staining, excluding the nucleoli, are associated with less-condensed chromatin and with transcriptional activity (24). By contrast, regions with DAPI staining are associated with heterochromatin, which is highly condensed and is correlated with transcription-repressor proteins (24). A previous study demonstrated that SDC-1 functions as an inhibitor of HAT, which is associated with transcriptional 
activity (13), suggesting that nuclear SDC-1 location could be associated with chromatin compaction. According to the ID-SDC-1/DAPI co-localization results from the present study, the majority of ID-SDC-1 was located in the compacted chromatin area. In addition, SNAIL has been demonstrated to act as a regulator of heterochromatin domains, through the co-repressor Lysyl Oxidase Like 2, in mouse embryonic fibroblast pericentromeric domains (25). Therefore, SNAIL overexpression may be associated with high heterochromatin stabilization and may favor an increased probability of nuclear ID-SDC1 with DAPI co-localization. However, more detailed studies of co-localization of ID-SDC1 with heterochromatin markers such as histone $\mathrm{H} 3$ lysine 9-methylation or co-immunoprecipitation of heterochromatin sequences with ID-SDC-1 are required.

SNAIL-overexpressing cells exhibited increased nuclear ID-SDC-1 protein levels compared with cytoplasmic levels. This could be associated with an alternative translation initiation, like that described for HER2 intracellular domains, located in the cytoplasm and nucleus (23).

Although EMT has been associated with the nuclear location of other proteins such as E-cadherin in other cancer types $(26,27)$, at present, the association between EMT factors and ID-SDC-1 location has not been described. In conclusion, the results of the present study demonstrated an association between SNAIL expression and nuclear ID-SDC-1 location in PCa cell lines.

The primary limitation of the present study is the low number of samples used for immunohistochemistry analyses (3 in each group). However, the statistical significance observed supports the conclusions concerning the expression and location of SNAIL, SLUG and ED-SDC-1. Nevertheless, a more extensive study is necessary for the clinical validation of these changes in the progression of $\mathrm{PCa}$.

\section{Acknowledgements}

The authors would like to thank to Mrs. Graciela Caroca (Department of Basic and Clinical Oncology, Faculty of Medicine, University of Chile, Santiago, Chile) for their technical assistance. The authors would also like to thank Dr María Julieta González, Dr Isabel Castro and Dr María José Barrera, from Biomedical Sciences Institute, University of Chile, for the confocal microscope use.

\section{Funding}

The present study was supported by grants from FONDECYT awarded to HRC (grant nos. 1110269 and 1151214) and to EAC (grant no. 1140417), Grants from U-APOYA ENLACE, University of Chile (grant nos. ENL-22/19 and ENL 23/19), State Research Agency and the European Regional Development Fund (grant no. SAF2016-76461-R) awarded to AGH and the CONICYT (National Commission of Science and Technology) scholarship (grant no. 21140772) awarded to NF.

\section{Availability of data and materials}

All data generated and analyzed during the current study are available from the corresponding author on reasonable request.

\section{Authors' contributions}

NF, AGdH, EAC and HRC conceived and designed the study. NF, OOS, PC, GM, DC and DH conducted the experiments and analyzed the data. NF, EAC and HRC wrote and revised the manuscript. All the authors read and approved the final manuscript.

\section{Ethics approval and consent to participate}

The protocol used for tissue collection was approved by Faculty of Medicine and CHUCh Ethics Committees. All patients provided written informed consent. All protocols and handling of hazardous materials were approved by the Faculty of Medicine of the University of Chile Risk and Biosecurity Unit.

\section{Patient consent for publication}

All patients provided written informed consent.

\section{Competing interests}

The authors declare that they have no competing interests.

\section{References}

1. Bray F, Ferlay J, Soerjomataram I, Siegel RL, Torre LA and Jemal A: Global cancer statistics 2018: GLOBOCAN estimates of incidence and mortality worldwide for 36 cancers in 185 countries. CA Cancer J Clin 68: 394-424, 2018

2. Nieto MA, Huang RY, Jackson RA and Thiery JP: EMT: 2016. Cell 166: 21-45, 2016.

3. Micalizzi DS, Farabaugh SM and Ford HL: Epithelialmesenchymal transition in cancer: Parallels between normal development and tumor progression. J Mammary Gland Biol Neoplasia 15: 117-134, 2010.

4. Puisieux A, Brabletz T and Caramel J: Oncogenic roles of EMT-inducing transcription factors. Nat Cell Biol 16: 488-494, 2014.

5. Poblete CE, Fulla J, Gallardo M, Muñoz V, Castellón EA, Gallegos I and Contreras HR: Increased SNAIL expression and low syndecan levels are associated with high Gleason grade in prostate cancer. Int J Oncol 44: 647-654, 2014.

6. Contreras HR, Ledezma RA, Vergara J, Cifuentes F, Barra C, Cabello P, Gallegos I, Morales B, Huidobro C and Castellón EA: The expression of syndecan- 1 and -2 is associated with Gleason score and epithelial-mesenchymal transition markers, E-cadherin and beta-catenin, in prostate cancer. Urol Oncol 28: 534-540, 2010.

7. Ledezma R, Cifuentes F, Gallegos I, Fullá J, Ossandon E, Castellon EA and Contreras HR: Altered expression patterns of syndecan-1 and -2 predict biochemical recurrence in prostate cancer. Asian J Androl 13: 476-480, 2011.

8. Dhodapkar MV, Abe E, Theus A, Lacy M, Langford JK, Barlogie B and Sanderson RD: Syndecan-1 is a multifunctional regulator of myeloma pathobiology: Control of tumor cell survival, growth, and bone cell differentiation. Blood 91: 2679-2688, 1998.

9. Leppä S, Mali M, Miettinen HM and Jalkanen M: Syndecan expression regulates cell morphology and growth of mouse mammary epithelial tumor cells. Proc Natl Acad Sci USA 89: 932-936, 1992.

10. Hu Y, Sun H, Owens RT, Gu Z, Wu J, Chen YQ, O'Flaherty JT and Edwards IJ: Syndecan-1-dependent suppression of PDK1/Akt/bad signaling by docosahexaenoic acid induces apoptosis in prostate cancer. Neoplasia 12: 826-836, 2010.

11. Tumova S, Woods A and Couchman JR: Heparan sulfate proteoglycans on the cell surface: Versatile coordinators of cellular functions. Int J Biochem Cell Biol 32: 269-288, 2000. 
12. Brockstedt $U$, Dobra $K$, Nurminen $M$ and Hjerpe $A$ : Immunoreactivity to cell surface syndecans in cytoplasm and nucleus: Tubulin-dependent rearrangements. Exp Cell Res 274: 235-245, 2002.

13. Purushothaman A, Hurst DR, Pisano C, Mizumoto S, Sugahara K and Sanderson RD: Heparanase-mediated loss of nuclear syndecan-1 enhances histone acetyltransferase (HAT) activity to promote expression of genes that drive an aggressive tumor phenotype. J Biol Chem 286: 30377-30383, 2011.

14. Chen L and Sanderson RD: Heparanase regulates levels of syndecan-1 in the nucleus. PLoS One 4: e4947, 2009.

15. Zong F, Fthenou E, Wolmer N, Hollósi P, Kovalszky I, Szilák L, Mogler C, Nilsonne G, Tzanakakis G and Dobra K: Syndecan-1 and FGF-2, but not FGF receptor-1, share a common transport route and co-localize with heparanase in the nuclei of mesenchymal tumor cells. PLoS One 4: e7346, 2009.

16. Szatmári T and Dobra K: The role of syndecan-1 in cellular signaling and its effects on heparan sulfate biosynthesis in mesenchymal tumors. Front Oncol 3: 310, 2013.

17. Stewart MD, Ramani VC and Sanderson RD: Shed syndecan-1 translocates to the nucleus of cells delivering growth factors and inhibiting histone acetylation: A novel mechanism of tumor-host cross-talk. J Biol Chem 290: 941-949, 2015.

18. Bernfield M, Götte M, Park PW, Reizes O, Fitzgerald ML, Lincecum $\mathbf{J}$ and Zako M: Functions of cell surface heparan sulfate proteoglycans. Annu Rev Biochem 68: 729-777, 1999.

19. Kovalszky I, Hjerpe A and Dobra K: Nuclear translocation of heparan sulfate proteoglycans and their functional significance. Biochim Biophys Acta 1840: 2491-2497, 2014.

20. Farfán N, Ocarez N, Castellón EA, Mejía N, de Herreros AG and Contreras HR: The transcriptional factor ZEB1 represses Syndecan 1 expression in prostate cancer. Sci Rep 8: 11467, 2018.
21. Herrera D, Orellana-Serradell O, Villar P, Torres MJ, Paciucci R, Castellón EA and Contreras HR: Silencing of the transcriptional factor ZEB1 alters the steroidogenic pathway, and increases the concentration of testosterone and DHT in DU145 cells. Oncol Rep 41: 1275-1283, 2019.

22. Fortini ME: Gamma-secretase-mediated proteolysis in cell-surface-receptor signalling. Nat Rev Mol Cell Biol 3: 673-684, 2002.

23. Anido J, Scaltriti M, Bech Serra JJ, Santiago Josefat B, Todo FR, Baselga $J$ and Arribas J: Biosynthesis of tumorigenic HER2 C-terminal fragments by alternative initiation of translation. EMBO J 25: 3234-3244, 2006.

24. Solovei I, Thanisch K and Feodorova Y: How to rule the nucleus: Divide et impera. Curr Opin Cell Biol 40: 47-59, 2016.

25. Millanes-Romero A, Herranz N, Perrera V, Iturbide A, Loubat-Casanovas J, Gil J, Jenuwein T, García de Herreros A and Peiró S: Regulation of heterochromatin transcription by Snail1/LOXL2 during epithelial-to-mesenchymal transition. Mol Cell 52: 746-757, 2013.

26. Céspedes MV, Larriba MJ, Pavón MA, Alamo P, Casanova I, Parreño M, Feliu A, Sancho FJ, Muñoz A and Mangues R: Site-dependent E-cadherin cleavage and nuclear translocation in a metastatic colorectal cancer model. Am J Pathol 177: 2067-2079, 2010

27. Chetty R, Serra S and Asa SL: Loss of membrane localization and aberrant nuclear E-cadherin expression correlates with invasion in pancreatic endocrine tumors. Am J Surg Pathol 32: 413-419, 2008.

This work is licensed under a Creative Commons Attribution-NonCommercial-NoDerivatives 4.0 International (CC BY-NC-ND 4.0) License. 